\title{
FUNDAMENTAL PARAMETERS : NORMAL AO DWARF STARS OF THE SOLAR NEIGHBOURHOOD
}

\author{
M. GERBALDI \\ CNRS and Université de Paris Sud XI \\ Institut d'Astrophysique 98bis, Bd. Arago, 75014 Paris, France \\ R. FARAGGIANA \\ Dipartimento di Astronomia v. Tiepolo 11, 34131 Trieste, Italy \\ R. BURNAGE \\ CNRS, Observatoire de Haute Provence 04870 Saint Michel l'Observatoire, France \\ F. DELMAS \\ CNRS Institut d'Astrophysique 98bis, Bd. Arago, 75014 Paris, France \\ A. GOMEZ \\ DASGAL, Observatoire de Meudon, 92150 Meudon, France \\ AND \\ S. GRENIER \\ DASGAL, Observatoire de Meudon, 92150 Meudon, France
}

\section{Introduction}

The purpose of this paper is to define a set of "standard", "reference" or "comparison" stars, according to their use, of spectral type A0V in order to be used for further studies in the domain of peculiar stars as well as normal stars of this $\mathrm{T}_{\text {eff }}$ and luminosity range.

Among the early type stars in the solar neighbourhood the A0 dwarfs are the hottest for which a significant number of objects is available. The aim of this study is to determine the atmospheric parameters necessary to put them on the $H R$ diagramme, the $\mathrm{M}_{V}$ being the value obtained from the HIPPARCOS parallaxes.

\section{Selection of the sample}

The sample was selected from the Bright Stars Catalogue (1982 edition), it consists of all the stars with a spectral type A0V excluding the stars recognized in this catalogue as being peculiar, shell stars or SB2. The papers by Abt and Morrell (1995) and Gray and Garrison (1987) have been scrutinized as well as the notes in the BSC in order to detect any quoted peculiarity. The final sample contains 230 objects.

\section{Atmospheric Parameters}

For such a set of stars, the best way to determine $T_{e \int f}$ and $\log g$ is from photometry and for early-type stars the uvbyH $\beta$ and Geneva are the most commonly used systems.

For the uvbyH $\beta$ system we shall use the classical calibration of the photometric indices in terms of $\mathrm{T}_{\text {eff }}, \log \mathrm{g}$ by Moon and Dworetsky (1985) (noted hereafter MD) which has been testerd ancl refined since more than a decade ago.

For the Geneva system, we took the calibration published by Künzli et al. (1997). The calibration of the Geneva photometric system is quite independent of the Strömgren one in the sense that it does not include any narrow band centered on a Balmer line; it is therefore interesting to compare 


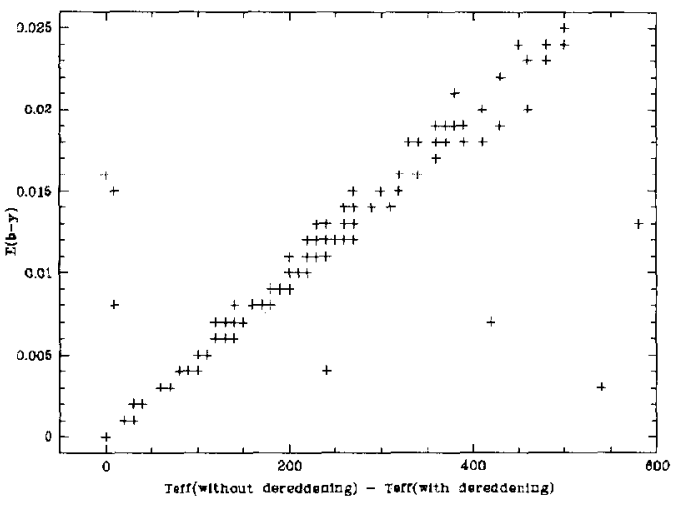

Figure 1. The effect of a moderate color excess on $\mathbf{T}_{\text {eff }}$ determination.

the results obtained with these two systems.

A final check of the reliability of these photometrically derived parameters can be done by comparing the observed spectra of a sub-sample of these stars with the synthetic spectra computed from models using as input data the photometrically determined parameters. The recent literature has widely demonstrated the validity of the computed spectra based on the last version of the Kurucz (1993) models.

We recall that as a first step dereddened colors must be computed; for our set of data de-reddening is expected to be very low or negligible; a severe reddening is likely to indicate a flux distortion due to spectral peculiarities or undetected binarity. The color excess $\mathrm{E}(\mathrm{b}-\mathrm{y})$ has been computed using Moon (1985) program UVBYBETA.

Slightly more than 140 stars have a color excess in the range : $-0.015 \leq \mathrm{E}(\mathrm{b}-\mathrm{y}) \leq+0.015$.

For all the other stars having an $\mathrm{E}(\mathrm{b}-\mathrm{y})$ value either negative or positive, we have looked at their bibliography and found that in many cases, about $40 \%$ of the sample, some peculiarities or binarity have been detected affecting the values of the color indices and therefore the computed color-excess.

\section{Influence of the value of $\mathbf{E}(\mathbf{b}-\mathbf{y})$ on $\mathbf{T}_{\text {eff }}$, $\log \mathbf{g}$}

For the stars with $\mathrm{E}(\mathrm{b}-\mathrm{y}) \geq 0.0$ we computed Teff and $\log \mathrm{g}$ assuming $\mathrm{E}(\mathrm{b}-\mathrm{y})=0$ and we plotted the difference of the $\mathrm{T}_{\text {eff }}$ derived with and without dereddening of color indices, versus $\mathrm{E}(\mathrm{b}-\mathrm{y})$ in Fig.1.

As expected a mean linear relation is found and the scatter around the mean value increases with the color excess. It is important to underline that the introduction of a moderate reddening of $\mathrm{E}(\mathrm{b}-\mathrm{y})=0.01$ produces a difference in $\mathrm{T}_{\text {eff }}$ of $200 \mathrm{~K}$.

Some stars lie completely outside from this mean relation : the value of their color indices was outside the limits of the grid used by MD.

The effect of a moderate reddening is negligible on the $\log g$ determination (Fig.2)

\section{Comparison between $\mathbf{T}_{\text {eff }}$ and $\log \mathrm{g}$ determined from uvbyH $\beta$ and Geneva Photometry}

For stars with $-0.015 \leq \mathrm{E}(\mathrm{b}-\mathrm{y}) \leq+0.015$, we have compared the values, in each photometric system, the value of $\mathrm{E}(\mathrm{b}-\mathrm{y})$ was taken as 0.0 . The $\mathrm{T}_{e f f}$ determined by the calibration of $\mathrm{MD}$ is systematically higher than that from the Geneva photometry. The mean value of the difference is $70 \mathrm{~K}$ ( $\mathrm{rms}=137 \mathrm{~K}$ ). There is a systematic effect on $\log \mathrm{g}$ in the sense that for $\log \mathrm{g} \leq 3.9$ the value com- 


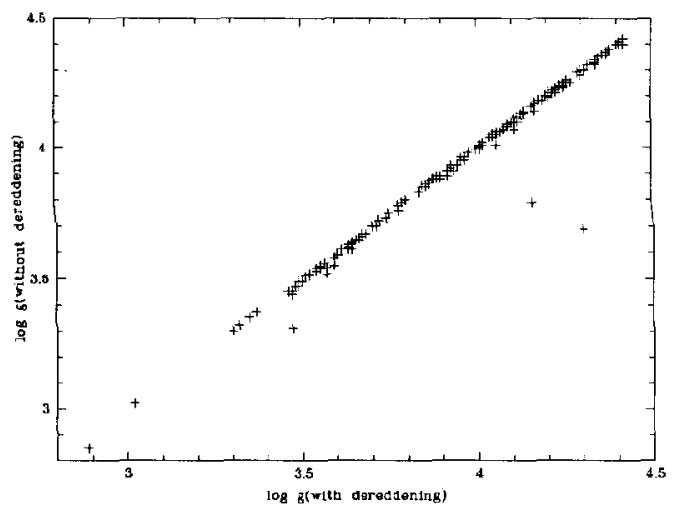

Figure 2. The relation between $\log g$ computed with a correction of the color excess and without correction.

puted from Geneva photometry is higher than that from $\mathrm{MD}$ and it is the reverse for $3.9 \leq \log \mathrm{g}$; all these differences are within 0.2 in $\log g$.

\section{The HR diagramme}

We found that even with a carefully selected sample from the BS Catalogue, we may still discover peculiar stars using spectroscopic data. To plot the HR diagramme for AOV stars we restricted our sample to all the stars for which we had obtained spectra at resolution of 28000 with the Echelec spectrograph at the ESO $1.52 \mathrm{~m}$ telescope. Among the 70 stars so far observed, 50 remain with a "normal" spectrum that is, with no obvious duplicity or any kind of peculiarity.

Fig. 3 shows the HR diagramme : in abscissa is plotted $\mathrm{T}_{\text {eff }}$ derived from the uvbyH $\beta$ system, and $M_{V}$ is from the HIPPARCOS parallax data. The error on $\mathrm{M}_{V}$ for all these stars is less than 0.3 mag..

The evolutionary tracks of Claret and Giménez (1992) are overlapped in Fig.3.

On this diagramme we note the spread of the points over a large range of $M_{V}$ which points out the difficulty to assign a luminosity class in this range of $\mathrm{T}_{e f f}$ for dwarf stars.

According to the accuracy of $T_{e f f}$ and $M_{V}$ for this sample we note that it is possible to give a reliable age for field stars; stars as young as 7.5 in $\log$ (years) are present in the solar neighbourhood. 


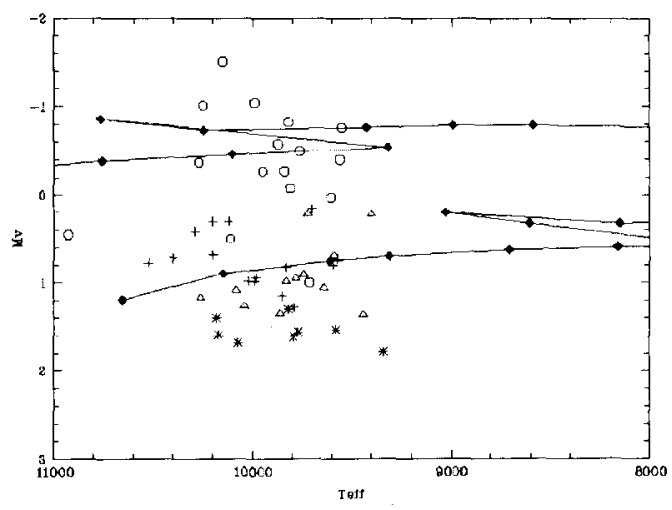

Figure 3. The HR diagramme; the upper evolutionary track is for $3.16 \mathrm{M} \Theta$, the lower for $2.51 \mathrm{M} \odot$. The various symbols correspond to the following values of the gravity : hexagon $: 3.3<\log \mathrm{g} \leq 3.8$; $\operatorname{cross}: 3.8<\log \mathrm{g} \leq 4.15$; triangle : $4.15<\log \mathrm{g} \leq 4.3 ;$ star $: 4.3<\log \mathrm{g} \leq 4.5$.

To conclude we put the emphasis on the number of binary stars that we found in this sample from our spectroscopic survey, the two members having similar spectral types. This binarity, when non detected produces a color excess which can be interpreted as a reddening and so is hampering the determination of $\mathrm{T}_{e f f}$ from any photometric calibration.

\section{References}

Abt, H.A. and Morrell, N.I. 1995, ApJS 99, 135

Claret, A. and Giménez, A. 1992, A\&AS, 96, 255

Gray, R.O. and Garrison, R.F. 1987, Ap.JS 65,581

Künzli, M., North, P., Kurucz, R.L., Nicolet, B. 1997, A\&AS, 122, 51

Kurucz, R.L. 1993, CD-Rom 18

Moon, T.T 1985, Comm. Univ. London Obs. 78, 1

Moon, T.T and Dworetsky, M.M. 1985, MNRAS 217,305 